\title{
In Silico Design, Synthesis, Anti-Microbial and Anti- Tubercular Evaluation of New Series of Benzothiazinone Derivatives Containing Triazolo Thiadiazole Moiety
}

Paridhi Jaiswal ( $\square$ paridhi.jaiswal@medicaps.ac.in )

Medi-Caps University https://orcid.org/0000-0002-1140-8672

Neha Kawathekar

Institute of Technology and Science

Gourav Jain

Institute of Technology and Science

Sanjay Jain

Medi-Caps University

\section{Research Article}

Keywords: Tuberculosis, DprE1 enzyme, Docking analysis, Benzothiazinone, Anti-microbial activity, Antitubercular activity.

Posted Date: July 14th, 2021

DOI: https://doi.org/10.21203/rs.3.rs-688359/v1

License: (c) (1) This work is licensed under a Creative Commons Attribution 4.0 International License.

Read Full License 


\title{
Title page
}

\section{In Silico Design, Synthesis, Anti-Microbial and Anti-Tubercular Evaluation of New Series of Benzothiazinone Derivatives Containing Triazolo Thiadiazole Moiety}

\author{
Ms. Paridhi Jaiswal ${ }^{1 *}$, Dr. Neha Kawathekar ${ }^{2}$, Mr. Gourav Jain ${ }^{3}$, Dr Sanjay Jain ${ }^{4}$ \\ Department of Pharmacy, Shri G.S. Institute of Technology and Sciences, Indore, Madhya \\ Pradesh, India, 452001.
}

\section{Author Names \& Affiliations:}

Ms. Paridhi Jaiswal* - Assistant Professor, Faculty of Pharmacy, Medi-Caps University, Indore

Dr. Neha Kawathekar - Head of the Department, Shri G.S. Institute of Technology and Science, Indore. She helped in Conceptualization, Conducting a research and investigation process, performing the experiments, data/evidence collection, Programming of Schrodinger Software for Molecular Docking.

Mr. Gourav Jain - Assistant Professor, Shri G.S. Institute of Technology and Science, Indore. Development \& Design of Methodology, Visualization, Validation, Literature review search, Preparation of Research article.

Dr Sanjay Jain - Dean, Faculty of Pharmacy, Medi-Caps University, Indore. Data Curation, analysis and interpretation of the data related to experimental work.

Corresponding Author: Ms. Paridhi Jaiswal (Assistant Professor, Faculty of Pharmacy, MediCaps University, Indore)

Add: 116/118 Suyash Vihar, Nanda Nagar, Indore (M.P.) India, 452011.

Email-paridhi.jaiswal19@gmail.com,paridhi.jaiswal@medicaps.ac.in

Phone number- 7024802363, 9098272668 


\begin{abstract}
Tuberculosis has proved harmful to the entire history of mankind from past several decades. Among the strategies for the development of new anti-tubercular lead compounds, Benzothiazinone (BTZ) were studied, with highly selective mechanism of action on DprE1 (Decaprenyl phosphoryl-b-D-ribose 2-epimerase) flavoenzyme. DprE1 is a vital enzyme for cell wall synthesis, plays a crucial role in the formation of D-arabinofuranose, a component of lipoarabinomannan and arabinogalactam. Formation of covalent and non covalent bond by the interacting ligand with the enzyme causes loss of its catalytic activity which ultimately leads to death of the mycobacterium.
\end{abstract}

The Benzothiazinone derivatives kill the mycobacterium by inhibiting the essential flavoenzyme DprE1. This study involves designing of a series of 16 Benzothiazinone derivatives that binds non covalently to DprE1. These derivatives were subjected to energy minimization and molecular docking studies with Schrodinger software, from which the binding free energy calculations showed that the suggested compounds had better binding affinity with DprE1. Ten compounds were selected for synthesis on the basis of docking score. The synthesized compounds were characterized and evaluated for their antimicrobial and anti-tubercular activity against different strains. Out of 5 evaluated compounds 3 compounds exhibited potent activity against microbial (Staphylococcus Aureus and Escherichia Coli) and tubercular (H37RV) strains.

BTZ4 and BTZ10 has shown good activity against anti-microbial strain and BTZ8 has shown good activity against anti-tubercular strain. On the basis of docking results, we concluded that the selected new series of Benzothiazinone hybrid derivatives BTZ4, BTZ7, BTZ8, BTZ9, BTZ10 act on the Enzyme DprE1, against tuberculosis binding protein with PDB ID: 4NCR, as they may have better binding sites and better energy values, when compared with the standard drugs Ampicillin, Isoniazid and Rifampicin. So these derivatives (BTZ4, BTZ8, BTZ10) can be taken as best hit molecule and it could be useful for development of more new antimicrobial and anti-tubercular agents, blocking the mycobacterial cell wall formation.

Keywords: Tuberculosis, DprE1 enzyme, Docking analysis, Benzothiazinone, Anti-microbial activity, Anti-tubercular activity. 


\section{Introduction}

\section{Tuberculosis}

Tuberculosis (TB) is an airborne contagious disease, caused by Mycobacterium bacilli or Mycobacterium tuberculosis (Mtb) that was discovered and isolated by Robert Koch in 1882. It is a rod-shaped bacillus of 1-4 $\mu \mathrm{m}$ length and 0.3-0.6 $\mu \mathrm{m}$ width. It generally attacks the lungs and is known as pulmonary tuberculosis. It is transmitted from person to person through droplets from cough or sneeze. It can also affect other organs, such as skeleton, soft tissue, lymph nodes and brain leading to extra pulmonary TB or it can disseminate through the blood vessels and affect multiple organs. It is the second reason for death on the planet from an infectious disease after human immunodeficiency infection (HIV).

They can be arranged into several major groups according to their treatment and diagnosis; alongside the previously mentioned Mycobacterium tuberculosis, additionally $M$. bovis, $M$. aficanum, M. bovis, M. caprae, M. canetti and M. microti have a place with the Mycobacterium tuberculosis complex (MTBC), and may cause TB diseases in humans and animals.

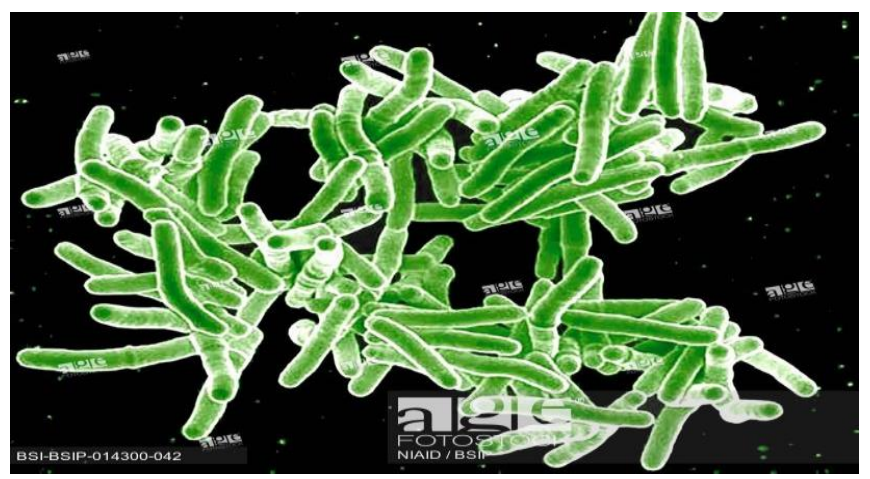

Figure 1: Scanning electron micrograph of Mycobacterium tuberculosis bacteria.

BTZ (Benzothiazinone) - A new class of anti-TB compounds having 1,3-benzothiazin-4-ones core (BTZs) has been recently disclosed. BTZ are the most promising, as it proved to kill Mycobacterium tuberculosis in vitro, ex vivo, and in a mouse models of TB infection and also responsible for blocking the synthesis of D-Arabinofuranose, a component of arabinogalactan and arabinomannan, with a mechanism of action highly selective in the cell wall of mycobacteria.

Benzothiazinones (BTZs), covalently binds to an active site with cysteine residue (Cys387 in $M$. tuberculosis, Cys394 in Mycobacterium smegmatis) in the essential enzyme DprE1, which 
catalyze biosynthesis of arabinose. Thus this enzyme losses its catalytic activity which ultimately leads to the death of mycobacteria. Thereby causing quantitative and irreversible inactivation.

Benzothiazinone inhibit the conversion of decaprenylphosphoryl- $\beta$-D-ribose(DPR) to decaprenylphosphoryl- $\beta$-D-arabinofuranose (DPA), a precursor of mycobacterial cell wall arabinan. This two-step epimerization reaction is catalyzed by the joint or successive action of the FAD-containing decaprenylphosphoryl- $\beta$-D-ribose 2'-epimerase (DprE1 or, Rv3790) and the NADH-dependent reductase DprE2 (Rv3791). BTZ inhibits the DprE1, thus provoking cell lysis and bacterial death.

\section{Materials And Method}

\subsection{Experimental}

\section{General Structure}

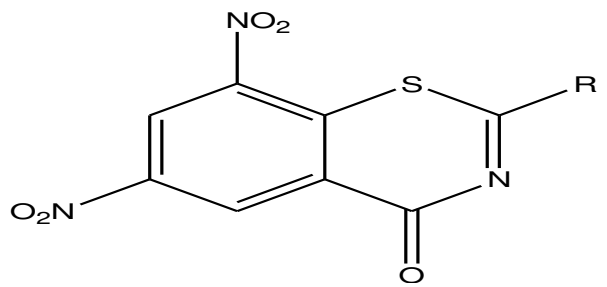

The present work addresses the synthesis of novel structural analogs of BTZ043. In particular, the chemical space at the arene moiety, introducing different substituents at positions 6 and 8 will be investigated. Furthermore, the influence of the position 2 substituent on BTZ activity will be investigated by introducing different cyclic amines as well as aryl and heteroaryl substituents.

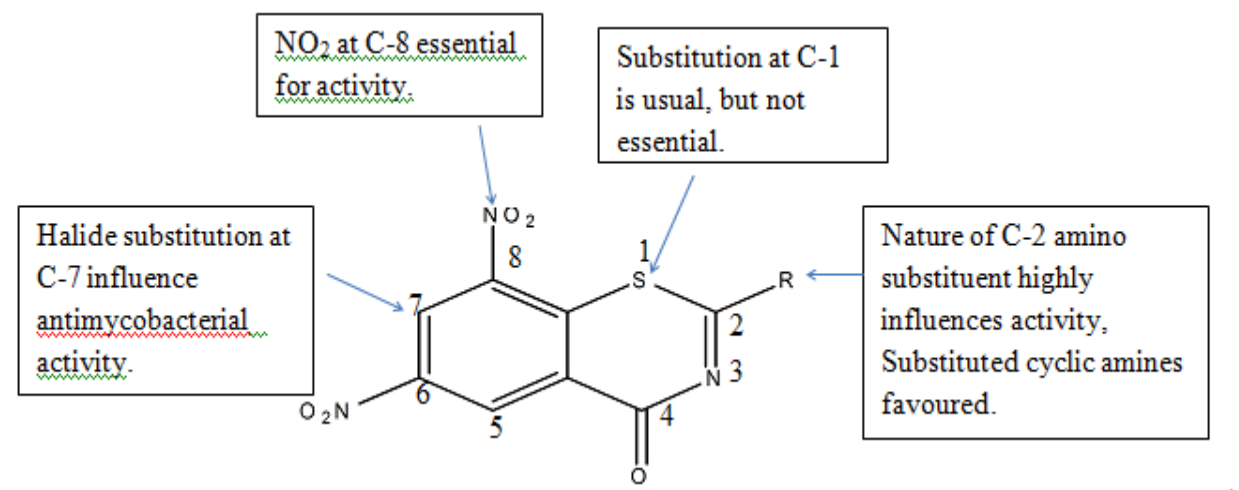

Chemicals and reagents were purchased from Alfa Aesar and Chem Pura Enterprises Pvt. Ltd. All reactions were monitored by thin layer chromatography (TLC) on silica gel plates using DCM:Methanol (9:1) as eluent while UV lamp was used to visualize the spot. Melting points were determined by open capillary method and are uncorrected. IR spectra of the synthesized 
compounds was recorded on IR AFFINITY-1 1400 using KBr pellet technique. ${ }^{1} \mathrm{H}$ NMR spectra were recorded using JNM-ECX500FT NMR spectrometers using DMSO as solvent. Chemical shifts are expressed in $\delta$ ppm.

\section{Reaction Scheme of Intermediate 1:}

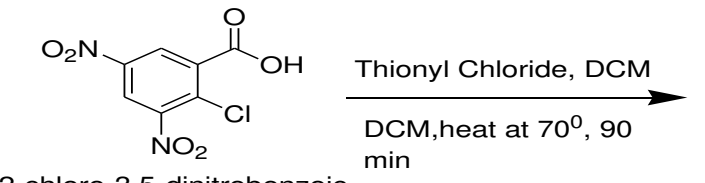<smiles>O=C(Cl)c1cc([N+](=O)[O-])cc([N+](=O)[O-])c1Cl</smiles>

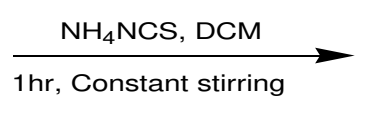

2-chloro-3,5-dinitrobenzoic acid

2-chloro-3,5-dinitrobenzoyl chloride<smiles>O=C(NS)c1cc([N+](=O)[O-])cc([N+](=O)[O-])c1Cl</smiles>

2-chloro-3,5-dinitrobenzoyl isothiocyanate

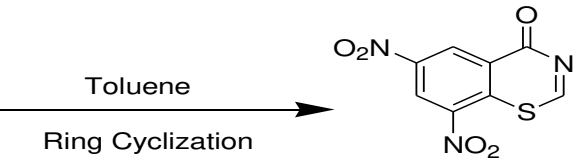

6,8-dinitro-4H-benzo[e][1,3]thiazin-4-one

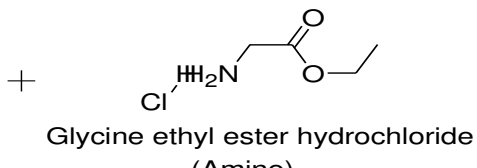

(Amine)

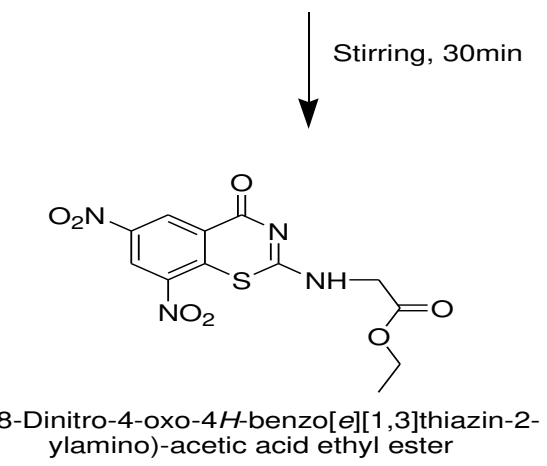

\section{Reaction Scheme of Intermediate 2:}

$$
\begin{aligned}
& \text { initro-4-oxo-4H-benzo[ } \mathrm{e}][1,3] \text { thiazin-2 } \\
& \text { ylamino)-acetic acid ethyl ester }
\end{aligned}
$$




\section{Reaction Scheme of Substitution:}

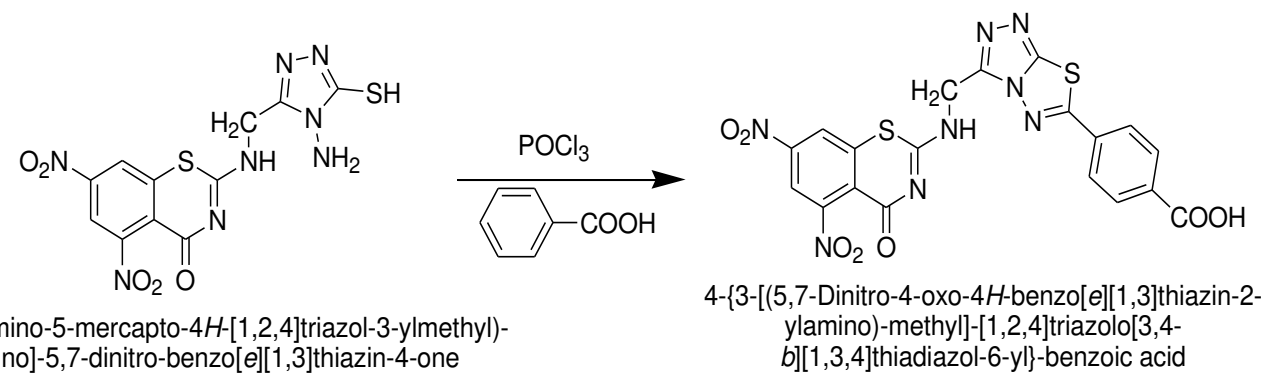

\subsection{General Procedure for synthesis}

\subsubsection{Synthesis of (6,8-Dinitro-4-oxo-4H-benzo[e][1,3]thiazin-2-ylamino)-acetic acid ethyl ester (Intermediate 1)}

To a RBF 2-Chloro-3,5-dinitro benzoic acid (2 g, 0.008 mole) in Dichloromethane (4-5 ml) with Thionyl chloride $(0.58 \mathrm{ml}, 0.004$ mole) and catalyst DMF $(2-3 \mathrm{ml})$ was added. The reaction mixture was heated at $70^{\circ} \mathrm{C}$ for 90 min. Clear solution was obtained and all the acid was consumed, then Ammonium thiocyanate ( $0.6 \mathrm{~g}, 0.001$ mole) was added portionwise during 10 min time interval with constant stirring for further $1 \mathrm{hr}$. Then Glycine ethyl ester $(1.1 \mathrm{~g}, 0.001$ mole) was added and stirring was continued for next $30 \mathrm{~min}$. The progress of reaction was monitored by TLC (DCM: Methanol 9:1) After completion of the reaction, the reaction mixture was mixed with saturated sodium bicarbonate solution and separated organic layer (DCM) was dried over anhydrous $\mathrm{MgSO}_{4}$. DCM was removed under reduced pressure. The desired product obtained as brown solid; M.P: $330-335^{\circ} \mathrm{C}$; yield: $94 \%$.

\subsubsection{Synthesis of 2-[(4-Amino-5-mercapto-4H-[1,2,4]triazol-3-ylmethyl)-amino]-5,7- dinitro-benzo[e][1,3]thiazin-4-one (Intermediate 2)}

To a RBF, the intermediate ester ( $2 \mathrm{~g}, 0.0056$ mole) was mixed with Hydrazine hydrate $(0.28 \mathrm{ml}$, $0.0091 \mathrm{~mole})$ in ethanol (2-3 ml) and stirring was done with reflux. To it, $\mathrm{KOH}(0.31 \mathrm{~g}, 0.0023$ mole) dissolved in ethanol, and then $\mathrm{CS}_{2}(0.52 \mathrm{ml}, 0.0091$ mole $)$ was added dropwise with stirring at room temperature and maintained for $12 \mathrm{hr}$. The salt was formed, filtered and washed with ethanol 3 times. Then the intermediate was dissolved in hot water $(2-3 \mathrm{ml})$ and Hydrazine hydrate $(0.17 \mathrm{ml}, 0.0068$ mole) was added. This mixture was heated and refluxed for $3 \mathrm{hr}$. After completion of the reaction, the reaction mixture was poured into ice water and acidified with 
conc. $\mathrm{HCl}$. The precipitate was filtered, washed with water. The desired product obtained as grey solid; M.P: $315-325^{\circ} \mathrm{C}$; yield: $83 \%$.

\subsubsection{Synthesis of 4-[3-(5,7-Dinitro-4-oxo-4H-benzo[e][1,3]thiazin-2-ylamino) $[1,2,4]$ triazolo $[3,4-b][1,3,4]$ thiadiazol-6-yl]-benzoic acid (BTZ1).}

In a $100 \mathrm{ml}$ RBF, a solution of intermediate (100 mg, 0.0027 mole) was taken, added Phosphoryl Chloride (0.3 ml, 0.0082 mole) dropwise. To it added Benzoic acid (0.2 g, 0.0048 mole) and the reaction mixture was stirred for $3 \mathrm{hrs}$ reflux. After completion of reaction, the reaction mixture was poured into ice water and $\mathrm{pH}$ was adjusted to 8.0 with $\mathrm{NaOH}$ solution. The ppt was formed, filtered and washed 3 times with ethanol. The desired product obtained as cream color solid; M.P: $280-285^{\circ} \mathrm{C}$; yield: $90 \%$.

\subsubsection{Synthesis of 2-[6-(2,4-Dichloro-phenyl)-[1,2,4]triazolo[3,4-b][1,3,4] thiadiazol-3- ylamino] -5,7-dinitro-benzo[e][1,3]thiazin-4-one (BTZ2).}

In a $100 \mathrm{ml}$ RBF, a solution of intermediate (100 mg, 0.0027 mole) was taken, added Phosphoryl Chloride (0.3 ml, 0.0082 mole) dropwise. To it added 2,4-dichloro benzoic acid (0.2 g, 0.0047 mole) and the reaction mixture was stirred for $3 \mathrm{hrs}$ reflux. After completion of reaction, the reaction mixture was poured into ice water and $\mathrm{pH}$ was adjusted to 8.0 with $\mathrm{NaOH}$ solution. The ppt was formed, filtered and washed 3 times with ethanol. The desired product obtained as yellow solid; M.P: $320-328^{\circ} \mathrm{C}$; yield: $88 \%$.

\subsubsection{Synthesis of 2-[6-(3,4-Dihydroxy-phenyl)-[1,2,4]triazolo[3,4-b][1,3,4] thiadiazol- 3ylamino] -5,7-dinitro-benzo[e][1,3]thiazin-4-one (BTZ3).}

In a $100 \mathrm{ml}$ RBF, a solution of intermediate (100 mg, 0.0027 mole) was taken, added Phosphoryl Chloride (0.3 ml, 0.0082 mole) dropwise. To it added 3,4-dihydroxy benzoic acid (0.2 g, 0.0048 mole) and the reaction mixture was stirred for $3 \mathrm{hrs}$ reflux. After completion of reaction, the reaction mixture was poured into ice water and $\mathrm{pH}$ was adjusted to 8.0 with $\mathrm{NaOH}$ solution. The ppt was formed, filtered and washed 3 times with ethanol. The desired product obtained as brown solid; M.P: $245-260{ }^{\circ} \mathrm{C}$; yield: $92 \%$. 


\subsubsection{Synthesis of 2-[6-(4-Amino-phenyl)-[1,2,4]triazolo[3,4-b][1,3,4]thiadiazol-3- ylamino]-5,7-dinitro-benzo[e][1,3]thiazin-4-one (BTZ4).}

In a $100 \mathrm{ml}$ RBF, a solution of intermediate ( $100 \mathrm{mg}, 0.0027$ mole) was taken, added Phosphoryl Chloride ( $0.3 \mathrm{ml}, 0.0082$ mole) dropwise. To it added p-amino benzoic acid ( $0.2 \mathrm{~g}, 0.0047$ mole) and the reaction mixture was stirred for $3 \mathrm{hrs}$ reflux. After completion of reaction, the reaction mixture was poured into ice water and $\mathrm{pH}$ was adjusted to 8.0 with $\mathrm{NaOH}$ solution. The ppt was formed, filtered and washed 3 times with ethanol. The desired product obtained as cream color solid; M.P: $278-285^{\circ} \mathrm{C}$; yield: $91 \%$.

\subsubsection{Synthesis of 5,7-Dinitro-2-[6-(4-nitro-phenyl)-[1,2,4]triazolo[3,4-b][1,3,4] thiadiazol-3-ylamino]-benzo[e][1,3]thiazin-4-one (BTZ5).}

In a $100 \mathrm{ml} \mathrm{RBF}$, a solution of intermediate ( $100 \mathrm{mg}, 0.0027$ mole) was taken, added Phosphoryl Chloride ( $0.3 \mathrm{ml}, 0.0082 \mathrm{~mole})$ dropwise. To it added p-nitro benzoic acid $(0.2 \mathrm{~g}, 0.0047$ mole $)$ and the reaction mixture was stirred for 3 hrs reflux. After completion of reaction, the reaction mixture was poured into ice water and $\mathrm{pH}$ was adjusted to 8.0 with $\mathrm{NaOH}$ solution. The ppt was formed, filtered and washed 3 times with ethanol. The desired product obtained as yellow solid; M.P: $315-326^{\circ} \mathrm{C}$; yield: $50 \%$.

\subsubsection{Synthesis of 2-[6-(4-Chloro-phenyl)-[1,2,4]triazolo[3,4-b] [1,3,4] thiadiazol-3- ylamino]-5,7-dinitro-benzo[e][1,3]thiazin-4-one (BTZ6).}

In a $100 \mathrm{ml} \mathrm{RBF}$, a solution of intermediate ( $100 \mathrm{mg}, 0.0027$ mole) was taken, added Phosphoryl Chloride ( $0.3 \mathrm{ml}, 0.0082$ mole) dropwise. To it added p-chloro benzoic acid ( $0.2 \mathrm{~g}, 0.0048$ mole) and the reaction mixture was stirred for $3 \mathrm{hrs}$ reflux. After completion of reaction, the reaction mixture was poured into ice water and $\mathrm{pH}$ was adjusted to 8.0 with $\mathrm{NaOH}$ solution. The ppt was formed, filtered and washed 3 times with ethanol. The desired product obtained as brown solid; M.P: $364-376^{\circ} \mathrm{C}$; yield: $84 \%$.

\subsubsection{Synthesis of 2-[6-(3-Amino-phenyl)-[1,2,4]triazolo[3,4-b][1,3,4]thiadiazol-3- ylamino]-5,7-dinitro-benzo[e][1,3]thiazin-4-one (BTZ7).}

In a $100 \mathrm{ml}$ RBF, a solution of intermediate ( $140 \mathrm{mg}, 0.0036$ mole) was taken, added Phosphoryl Chloride ( $0.36 \mathrm{ml}, 0.0099$ mole) dropwise. To it added m-amino benzoic acid $(0.23 \mathrm{~g}, 0.0082$ 
mole) and the reaction mixture was stirred for $3 \mathrm{hrs}$ reflux. After completion of reaction, the reaction mixture was poured into ice water and $\mathrm{pH}$ was adjusted to 8.0 with $\mathrm{NaOH}$ solution. The ppt was formed, filtered and washed 3 times with ethanol. The desired product obtained as light brown solid; M.P: $375-382{ }^{\circ} \mathrm{C}$; yield: $89 \%$.

\subsubsection{Synthesis of 2-[6-(2-Chloro-5-nitro-phenyl)-[1,2,4]triazolo[3,4-b][1,3,4] thiadiazol-3-ylamino]-5,7-dinitro-benzo[e][1,3]thiazin-4-one (BTZ8).}

In a $100 \mathrm{ml}$ RBF, a solution of intermediate (130 mg, 0.0034 mole) was taken, added Phosphoryl Chloride (0.35 ml, 0.0097 mole) dropwise. To it added 2-Chloro,5-nitro benzoic acid (0.21 g, 0.0078 mole) and the reaction mixture was stirred for $3 \mathrm{hrs}$ reflux. After completion of reaction, the reaction mixture was poured into ice water and $\mathrm{pH}$ was adjusted to 8.0 with $\mathrm{NaOH}$ solution. The ppt was formed, filtered and washed 3 times with ethanol. The desired product obtained as light brown solid; M.P: $266-274^{\circ} \mathrm{C}$; yield: $50 \%$.

\subsubsection{Synthesis of 2-[6-(2-Chloro-phenyl)-[1,2,4]triazolo[3,4-b][1,3,4]thiadiazol-3- ylamino]-5,7-dinitro-benzo[e][1,3]thiazin-4-one (BTZ9).}

In a $100 \mathrm{ml}$ RBF, a solution of intermediate (200 mg, 0.0054 mole) was taken, added Phosphoryl Chloride (0.6 ml, 0.0164 mole) dropwise. To it added 2-Chloro benzoic acid ( $0.4 \mathrm{~g}, 0.0096$ mole) and the reaction mixture was stirred for $3 \mathrm{hrs}$ reflux. After completion of reaction, the reaction mixture was poured into ice water and $\mathrm{pH}$ was adjusted to 8.0 with $\mathrm{NaOH}$ solution. The ppt was formed, filtered and washed 3 times with ethanol. The desired product obtained as brown solid; M.P: $392-395^{\circ} \mathrm{C}$; yield: $90 \%$.

\subsubsection{Synthesis of 5,7-Dinitro-2-[6-(2-nitro-phenyl)-[1,2,4]triazolo[3,4-b][1,3,4] thiadiazol-3-ylamino]-benzo[e][1,3]thiazin-4-one (BTZ10).}

In a $100 \mathrm{ml}$ RBF, a solution of intermediate ( $150 \mathrm{mg}, 0.0038$ mole) was taken, added Phosphoryl Chloride $(0.37 \mathrm{ml}, 0.0093 \mathrm{~mole})$ dropwise. To it added m-amino benzoic acid $(0.25 \mathrm{~g}, 0.0054$ mole) and the reaction mixture was stirred for $3 \mathrm{hrs}$ reflux. After completion of reaction, the reaction mixture was poured into ice water and $\mathrm{pH}$ was adjusted to 8.0 with $\mathrm{NaOH}$ solution. The ppt was formed, filtered and washed 3 times with ethanol. The desired product obtained as yellow solid; M.P: $255-268^{\circ} \mathrm{C}$; yield: $80 \%$. 
Table 1: Docking Score, Glide emodel energy and RMSD of (BTZ1- BTZ16).

\begin{tabular}{|c|c|c|c|c|}
\hline S.No. & Compound Code & Docking Score & Glide Emodel & RMSD \\
\hline 1 & BTZ1 & -8.893 & -85.792 & 0.049 \\
\hline 2 & BTZ2 & -6.528 & -67.850 & 0.043 \\
\hline 3 & BTZ3 & -7.063 & -85.101 & 0.034 \\
\hline $\mathbf{4}$ & BTZ4 & $\mathbf{- 9 . 5 6 9}$ & $\mathbf{- 9 9 . 1 8 5}$ & $\mathbf{0 . 0 4 2}$ \\
\hline 5 & BTZ5 & -6.928 & -71.258 & 0.059 \\
\hline 6 & BTZ6 & -8.282 & -82.318 & 0.028 \\
\hline $\mathbf{7}$ & BTZ7 & $\mathbf{- 9 . 1 6 2}$ & $\mathbf{- 8 4 . 9 1 4}$ & $\mathbf{0 . 0 3 8}$ \\
\hline $\mathbf{8}$ & BTZ8 & $\mathbf{- 9 . 1 4 8}$ & $\mathbf{- 9 2 . 6 1 7}$ & $\mathbf{0 . 0 4 2}$ \\
\hline $\mathbf{9}$ & BTZ9 & $\mathbf{- 9 . 0 4 9}$ & $\mathbf{- 9 5 . 0 3 1}$ & $\mathbf{0 . 0 0 5}$ \\
\hline 10 & BTZ10 & $\mathbf{- 5 . 7 8 2}$ & -66.879 & 0.045 \\
\hline 11 & BTZ11 & -6.246 & -66.249 & 0.041 \\
\hline 12 & BTZ12 & $\mathbf{- 5 . 6 8 5}$ & -65.165 & 0.017 \\
\hline 13 & BTZ13 & -6.755 & -78.841 & 0.034 \\
\hline 14 & BTZ14 & -6.300 & -68.734 & 0.046 \\
\hline $\mathbf{1 5}$ & BTZ15 & $\mathbf{- 9 . 1 7 6}$ & $\mathbf{- 9 4 . 1 1 1}$ & $\mathbf{0 . 0 2 3}$ \\
\hline 16 & BTZ16 & -5.345 & -65.023 & 0.015 \\
\hline 17 & Delamanid & $\mathbf{- 5 . 0 4 1}$ & 35.783 & 0.012 \\
\hline
\end{tabular}

\subsection{Characterization of Synthesized Compounds}

The identification and characterization of the prepared compounds were carried out by the following procedure to ascertain that the compounds were actually synthesized. Characterization was performed by the following methods:

\subsubsection{Thin Layer Chromatography:}

Thin Layer Chromatography is a method for analyzing mixtures by separating the compounds in the mixture. TLC can be used to determine the number of components in a mixture, the identity of compounds, and the purity of a compound. While observing the appearance of a product or the disappearance of a reactant, it can also be used to monitor the progress of a reaction. Thin Layer Chromatography was carried out with silica gel plates (silica gel $60 \mathrm{~F}_{254}$ ), and DCM:Methanol 
(9:1) or Chloroform:Ethanol (7:3) was used as mobile phase. $\mathrm{R}_{\mathrm{f}}$ value of synthesized compounds are reported in Table 2. The differences in $R_{f}$ values between starting compounds and the product were indicative of the transformation of starting compound to product and also purity of compounds.

Table 2: $\mathrm{R}_{\mathrm{f}}$ value with solvent system of synthesized compounds.

\begin{tabular}{|c|c|c|}
\hline Compound Code & Rf Value & Solvent System \\
\hline BTZ1 & 0.366 & Chloroform:Ethanol (7:3) \\
\hline BTZ2 & 0.697 & Chloroform:Ethanol (7:3) \\
\hline BTZ3 & 0.538 & Chloroform:Ethanol (7:3) \\
\hline BTZ4 & 0.363 & Ethyl acetate:Hexane(3:7) \\
\hline BTZ5 & 0.346 & Chloroform:Ethanol (7:3) \\
\hline BTZ6 & 0.560 & Chloroform:Ethanol (7:3) \\
\hline BTZ7 & 0.625 & Chloroform:Ethanol (7:3) \\
\hline BTZ8 & 0.304 & Chloroform:Ethanol (7:3) \\
\hline BTZ9 & 0.608 & Ethyl acetate:Hexane(3:7) \\
\hline BTZ10 & 0.426 & \\
\hline
\end{tabular}

\subsubsection{Melting Point:}

The melting point determination was done in melting point apparatus and are uncorrected. The melting points of all the synthesized compounds are reported in Table 3.

\subsection{3 cLogP values:}

The cLogPValues, the indicative of hydrophobicity, were predicted for all the derivatives using CS ChemOffice- 2004 version 8.0. The cLogP values are reported in Table 3.

Table 3: Molecular Formula, Molecular Weight, cLogP and Melting Point.

\begin{tabular}{|c|c|c|c|c|}
\hline $\begin{array}{c}\text { Compound } \\
\text { Code }\end{array}$ & $\begin{array}{c}\text { Molecular } \\
\text { Weight }\end{array}$ & $\begin{array}{c}\text { Molecular } \\
\text { Formula }\end{array}$ & cLogP & Melting Point \\
\hline BTZ1 & 512.44 & $\mathrm{C}_{18} \mathrm{H}_{8} \mathrm{~N}_{8} \mathrm{O}_{7} \mathrm{~S}_{2}$ & 2.599 & $280-285^{\circ} \mathrm{C}$ \\
\hline BTZ2 & 537.32 & $\mathrm{C}_{17} \mathrm{H}_{6} \mathrm{Cl}_{2} \mathrm{~N}_{8} \mathrm{O}_{5} \mathrm{~S}_{2}$ & 4.018 & $320-328^{\circ} \mathrm{C}$ \\
\hline BTZ3 & 500.42 & $\mathrm{C}_{17} \mathrm{H}_{8} \mathrm{~N}_{8} \mathrm{O}_{7} \mathrm{~S}_{2}$ & 1.663 & $245-260{ }^{\circ} \mathrm{C}$ \\
\hline BTZ4 & 483.44 & $\mathrm{C}_{17} \mathrm{H}_{9} \mathrm{~N}_{9} \mathrm{O}_{5} \mathrm{~S}_{2}$ & 1.655 & $278-285^{\circ} \mathrm{C}$ \\
\hline
\end{tabular}




\begin{tabular}{|c|c|c|c|c|}
\hline BTZ5 & 513.42 & $\mathrm{C}_{17} \mathrm{H}_{7} \mathrm{~N}_{9} \mathrm{O}_{7} \mathrm{~S}_{2}$ & 2.585 & $315-326^{\circ} \mathrm{C}$ \\
\hline BTZ6 & 502.87 & $\mathrm{C}_{17} \mathrm{H}_{7} \mathrm{ClN}_{8} \mathrm{O}_{5} \mathrm{~S}_{2}$ & 3.555 & $364-376{ }^{\circ} \mathrm{C}$ \\
\hline BTZ7 & 483.44 & $\mathrm{C}_{17} \mathrm{H}_{9} \mathrm{~N}_{9} \mathrm{O}_{5} \mathrm{~S}_{2}$ & 1.655 & $375-382{ }^{\circ} \mathrm{C}$ \\
\hline BTZ8 & 547.87 & $\mathrm{C}_{17} \mathrm{H}_{6} \mathrm{ClN}_{9} \mathrm{O}_{7} \mathrm{~S}_{2}$ & 3.048 & $266-274{ }^{\circ} \mathrm{C}$ \\
\hline BTZ9 & 502.87 & $\mathrm{C}_{17} \mathrm{H}_{7} \mathrm{ClN}_{8} \mathrm{O}_{5} \mathrm{~S}_{2}$ & 3.305 & $392-395{ }^{\circ} \mathrm{C}$ \\
\hline BTZ10 & 517.89 & $\mathrm{C}_{17} \mathrm{H}_{8} \mathrm{ClN}_{9} \mathrm{O}_{5} \mathrm{~S}_{2}$ & 2.372 & $255-268^{\circ} \mathrm{C}$ \\
\hline
\end{tabular}

\subsection{4 ${ }^{1}$ H NMR Spectroscopy:}

${ }^{1} \mathrm{H}$ NMR spectra were recorded using JNM-ECX500FTNMR spectrometers using DMSO as solvent at Dr. Harisingh Gour University, Sagar. ${ }^{1} \mathrm{H}$ NMR data of compounds are summarized in Table 4.

\subsubsection{IR Spectroscopy:}

The Infrared spectroscopy of all the synthesized compounds were recorded on IR AFFINITY-1 1400 using $\mathrm{KBr}$ pellet technique were carried out from Shreeji Analytical and Research Laboratory Pvt. Ltd, Indore and are expressed in $\mathrm{cm}^{-1}$. The IR data of the compounds are summarized in Table 4.

Table 4: ${ }^{1} \mathrm{H}$ NMR, and IR Prediction Values

\begin{tabular}{|c|c|c|}
\hline $\begin{array}{c}\text { Compound } \\
\text { Code }\end{array}$ & Interpretation of NMR (ppm) & Interpretation of $\operatorname{IR}\left(\mathrm{cm}^{1}\right)$ \\
\hline BTZ4 & & $\begin{array}{l}3350-3200 \mathrm{NH}_{2} \text { Bending,3000-2800 N- } \\
\text { HStretching, 2900-2800 C-HStretching, } \\
1690 \mathrm{C}=\mathrm{O} \text { Stretching, } \\
1600-1500 \text { N-OStretching. }\end{array}$ \\
\hline BTZ7 & & $\begin{array}{l}\text { 3450-3300 C-H Stretching, } 3400 \mathrm{~N}-\mathrm{H} \\
\text { Stretching,3200-3100 NH} 2 \text { Bending, } \\
\text { 2000-1500 C-H Bending, 1550-1500 N- } \\
\text { O Stretching, } 800 \text { C-H Bending, } 1650 \\
\text { C=O Stretching. }\end{array}$ \\
\hline
\end{tabular}




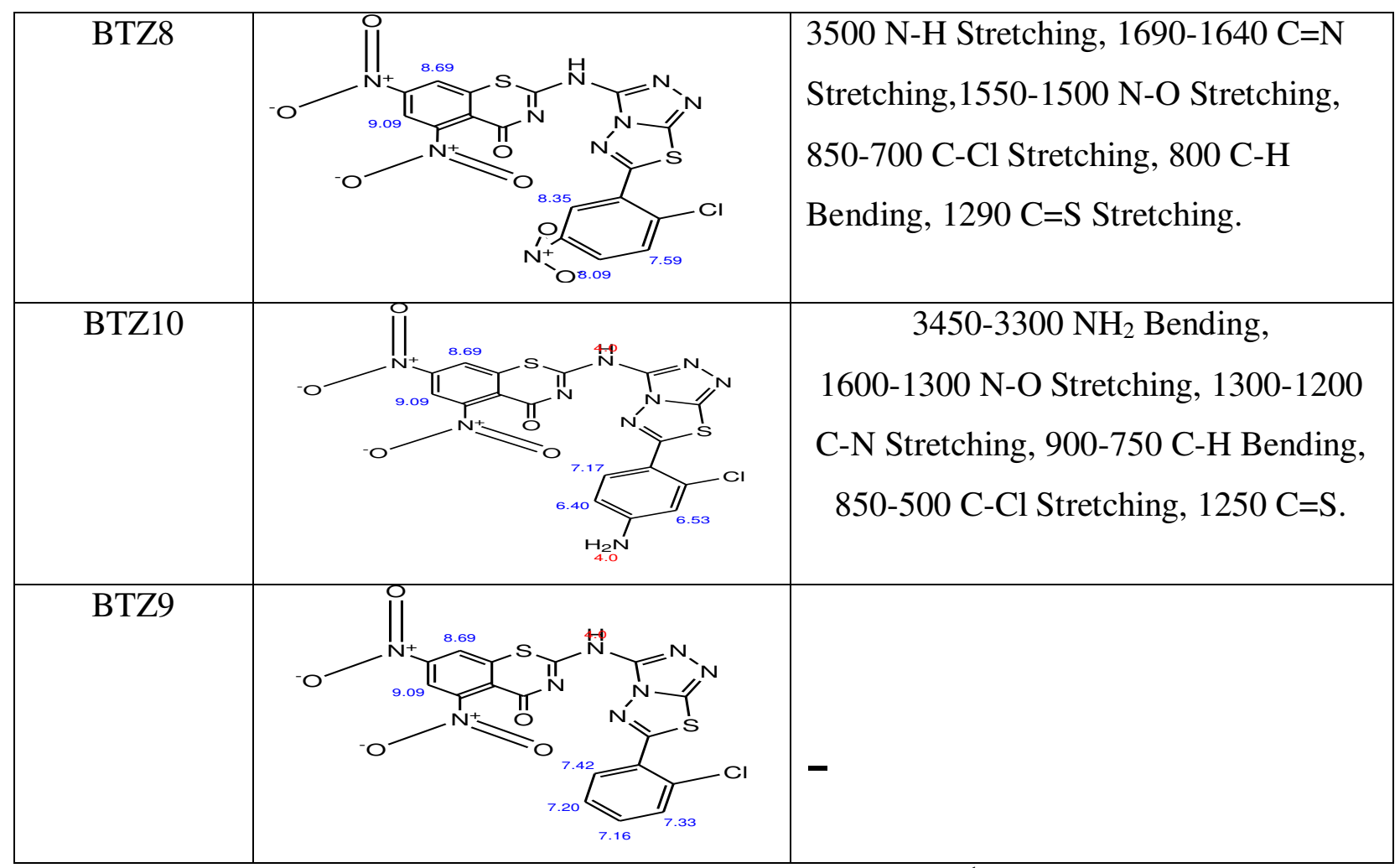

In the IR spectra, the relatively strong peaks at $3350-3200 \mathrm{~cm}^{-1}$ were attributed to the $\mathrm{NH}_{2}$ bending vibrations and the weak single peaks at $1600-1500 \mathrm{~cm}^{-1}$ due to the $\mathrm{N}-\mathrm{O}$ stretching vibrations for the compound BTZ4. However, in BTZ8, the relatively strong peaks at $3500 \mathrm{~cm}^{-1}$ were attributed to the $\mathrm{N}-\mathrm{H}$ stretching vibrations and the weak single peaks at $1550-1500 \mathrm{~cm}^{-1} \mathrm{due}$ to the $\mathrm{N}-\mathrm{O}$ stretching vibrations and $\mathrm{C}-\mathrm{Cl}$ stretching peaks at $850-700 \mathrm{~cm}^{-1}$

The ${ }^{1} \mathrm{H}$ NMR spectra of the Benzothiazinone derivative (BTZ4) showed two characteristic proton signals at 8.69 and $9.09 \mathrm{ppm}$, which were attributed to the protons of $\mathrm{N}-\mathrm{O}$ group and doublet proton signals at 7.23 and $6.52 \mathrm{ppm}$ which may be due to $\mathrm{C}-\mathrm{H}$ group. However, in BTZ8, the characteristic proton signal at 8.09 and $7.59 \mathrm{ppm}$ which may be due to $\mathrm{N}-\mathrm{O}$ and $\mathrm{C}-\mathrm{Cl}$ group respectively.

\subsection{Evaluation of Anti-microbial and Anti-tubercular Activity of Synthesized Compound}

\subsubsection{Anti-microbial Activity}

The antimicrobial activity was performed on human pathogenic bacteria (Staphylococcus aureus, Escherichia coli). In assaying antibiotic activity, by Cup borer method, the test organism was grown on a suitable complete agar medium in Petri dishes. "Cups" cut out of the agar were filled with appropriate dilutions of a standard compound solution and of the test compound. After 
incubation the cups are found to be surrounded by circular "Zone of inhibition". The Zone of inhibition was measured in terms of area in $\mathrm{mm}^{2}$ as shown in Table 5 and compared with the area of whole quadrant to find out 50\% inhibition compared with the standard drug Ampicillin.

Table 5: Zone of Inhibition of Synthesized Compound.

\begin{tabular}{|c|c|c|}
\hline Compound Code & Staphylococcus Aureus Gram (+) & Escherichia Coli Gram (-) \\
\hline BTZ4 & $17 \mathrm{~mm}$ & $19 \mathrm{~mm}$ \\
\hline BTZ7 & $21 \mathrm{~mm}$ & $24 \mathrm{~mm}$ \\
\hline BTZ10 & $18 \mathrm{~mm}$ & $16 \mathrm{~mm}$ \\
\hline Ampicillin & $24 \mathrm{~mm}$ & $22 \mathrm{~mm}$ \\
\hline
\end{tabular}

\subsubsection{Anti-tubercular activity}

\subsubsection{Culture}

Tubercle bacilli are aerobes, grow slowly (generation time 14-15 hrs). optimum temperature $37^{\circ} \mathrm{C}$, pH 6.4-7.0. They grow only in specially enriched media containing egg, asparagine, potatoes, serum and meat extracts. Colonies appear in 2-6 weeks. M. tuberculosis grows more luxuriantly in culture (eugenic) than M.bovis which grows sparsely (dysgenic). The drug susceptibility test may be performed by either the direct or the indirect method. The direct drug susceptibility test is performed by using a subculture from a primary culture as the inoculum.

\subsubsection{Determination of minimal inhibition concentrations by L.J Slope method.}

\section{Materials and Method}

1. All the synthesized drugs were used for anti-tubercle test procedures

2. All Necessary controls like:

Drug control

$>$ Vehicle control

Agar control

$>$ Organisms control

$>$ Known antibacterial drugs control

M.tuberculosis $\mathrm{H} 37 \mathrm{RV}$ cultures were tested against above mentioned known and unknown drugs. 
LJ was used as nutrient medium to grow and dilute the drug suspension for the test.

Inoculum size for test strain was adjusted to $1 \mathrm{mg} / \mathrm{ml}$.

Following common standard strain is used for screening of anti-tubercle activities: the strains were procured from institute of microbial technology, Chandigarh. Mycobacterium tuberculosis H37Rv (Acid Fast Bacilli) MTCC- 200 DMSO was as diluents/ Vehicle to get desired concentration of drugs to test upon Standard bacterial strains.

\subsubsection{Methods used for Primary and Secondary Screening:}

Each synthesized drug was diluted obtaining 2000 microgram/ml concentration, as a stock solution.

Primary screen: In primary screening $500 \mathrm{microgram} / \mathrm{ml}, 250 \mathrm{microgram} / \mathrm{ml}$ and 125 microgram $/ \mathrm{ml}$ concentrations of the synthesized drugs were taken. The active synthesized drugs found in this primary screening were further tested in a second set of dilution against all microorganisms.

Secondary screen: The drugs found active in primary screening were similarly diluted to obtain $100 \mu \mathrm{g} / \mathrm{ml}, 50 \mu \mathrm{g} / \mathrm{ml}, 25 \mu \mathrm{g} / \mathrm{ml}, 12.5 \mu \mathrm{g} / \mathrm{ml}, 6.250 \mu \mathrm{g} / \mathrm{ml}, 3.125 \mu \mathrm{g} / \mathrm{ml}$ and $1.5625 \mu \mathrm{g} / \mathrm{ml}$ concentrations.

Reading Result: The highest dilution showing at least $99 \%$ inhibition is taken as MIC.The result of this is much affected by the size of the inoculum. The test mixture should contain $10^{8}$ organism $/ \mathrm{ml}$.

Table 6: $\mathrm{IC}_{50}$ Values of synthesized compound for anti-tubercular activity.

\begin{tabular}{|l|l|l|l|}
\hline \multicolumn{2}{|c|}{ ANTITUBERCULOSIS ACTIVITY TABLE } \\
\hline METHOD & L.J. MEDIUM (CONVENTIONAL METHOD) \\
\hline BACTERIA & H37RV \\
\hline STANDARD DRUG & ISONIAZID, RIFAMPICIN \\
\hline S.NO & CODE NO & MIC $\boldsymbol{\mu g} / \mathbf{m l}$ & REMARKS \\
\hline
\end{tabular}




\begin{tabular}{|c|c|c|l|}
\hline 1 & BTZ8 & 0.28 & ISONIAZID $=0.39 \mu \mathrm{g} / \mathrm{ml}$ \\
\hline 2 & BTZ9 & 0.37 & RIFAMPICIN $=0.48 \mu \mathrm{g} / \mathrm{ml}$ \\
\hline
\end{tabular}

\section{Results and Discussion}

\subsection{Molecular docking}

The main aim of the present study is to identify and optimize leads of benzothiazinone derivatives against DprE1 as new antitubercular agents. Sixteen derivatives of benzothiazinone were designed. Glide score was obtained using GLIDE module (Grid based Ligand docking with Energetics, version Schrodinger 9.1, LLC, New York, 2010) at the CADD laboratory, S.G.S.I.T.S. Indore.

After preparation of ligand and protein, protein grid was generated by setting the grid box in which the ligands were expected to dock. Molecular docking of the designed compounds was performed to study the binding pattern of the structure with the protein. The docking studies were performed using protein (PDB ID; 4NCR)

Best docking scores of compounds were compared using docking score. The data shown in the given table revealed that all the designed compounds shows a prominent result as antitubercular and antimicrobial activity, but some compounds BTZ4, BTZ7, BTZ8, BTZ9, BTZ15 showed best Glide docking score as compared to other derivatives, which is compared with the Standard drugs (Ampicillin, Isoniazid and Rifampicin).

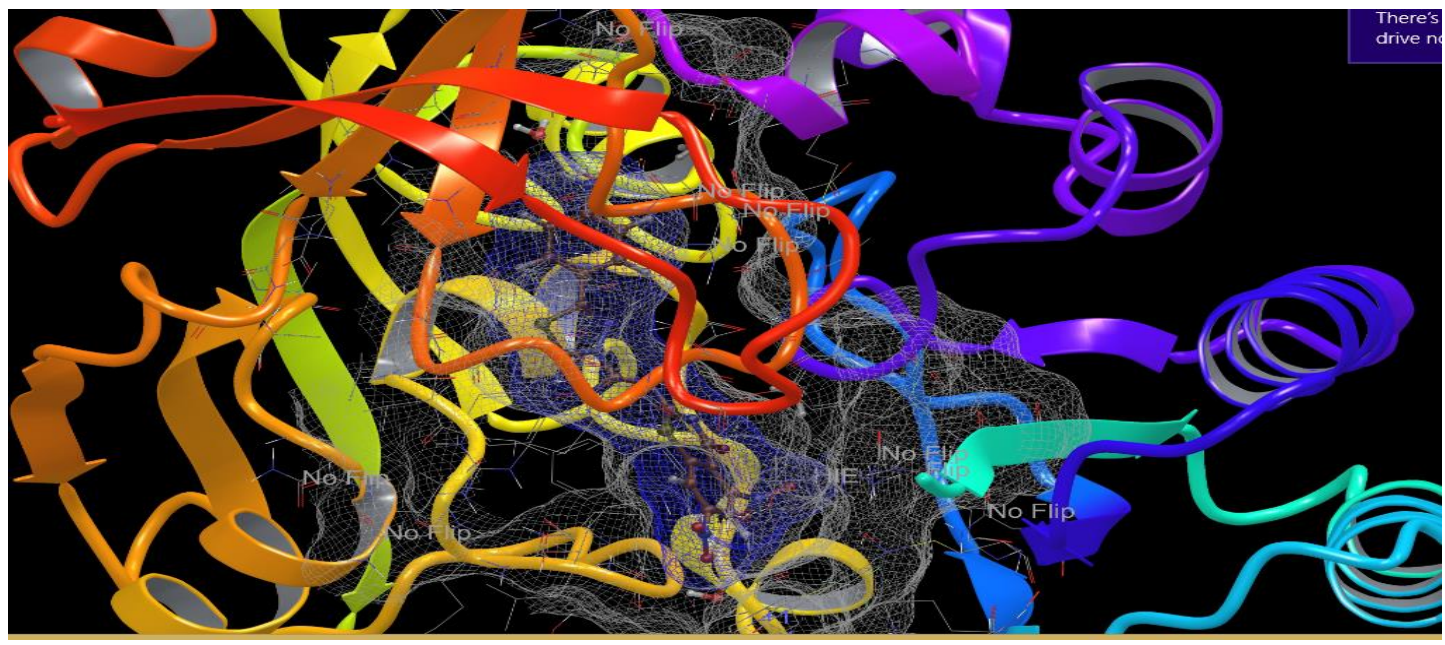

Figure 2: Best Binding Pose on Protein 4NCR of Compound (BTZ 4) 


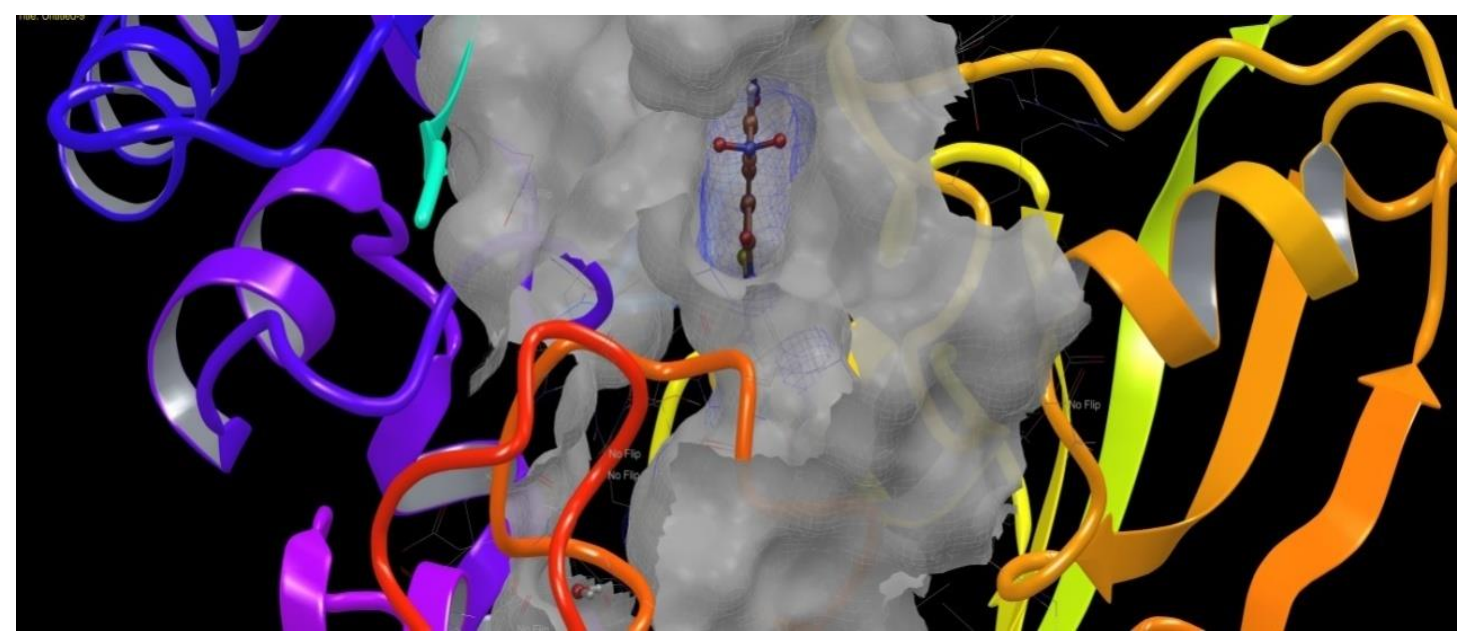

Figure 3: Best Binding Pose on Protein 4NCR of Compound (BTZ 7).

Structure based docking strategy was carried out using the poses predicted by Glide. All the ligands were docked within the binding site of the bacterial protein. Analysis of the docking result clarify that interaction of ligand with residue, is the crucial parameter for the inhibition of DprE1 (decaprenylphosphoryl- $\beta$-D-ribose 2'-epimerase) enzyme. Benzothiazinone derivatives form covalent or non-covalent binding with Cysteine C387 and Tyrosine Ty38c residue of the enzyme. In the molecular docking studies the compound BTZ 4 \& BTZ 7 has shown good interaction with protein residue and glide properties.

\subsection{Antimicrobial and Antitubercular activity}

The antimicrobial activity was performed on human pathogenic bacteria (Staphylococcus aureus, Escherichia coli). In assaying antibiotic activity by Cup borer method, the test organism was grown on a suitable complete agar medium in Petri dishes. "Cups" cut out of the agar were filled with appropriate dilutions of a standard compound solution and of the test compound. After incubation the cups are found to be surrounded by circular "Zone of inhibition". The Zone of inhibition was measured in terms of area in $\mathrm{mm}^{2}$ as shown in Table 5 and compared with the area of whole quadrant to find out 50\% inhibition compared with the standard drug Ampicillin.

Out of 5 evaluated compounds 3 compounds exhibited potent activity against microbial (Staphylococcus aureus and Escherichia coli) and tubercular (H37RV) strains. BTZ4 and BTZ10 has shown good activity against anti-microbial strain and BTZ8 has shown good activity against anti-tubercular strain. 


\section{Conclusion}

The main aim of the present study is to design various substituted benzothiazinone derivatives. On the basis of literature review, we envisaged that benzothiazinone derivatives have potentiated that non covalently react with the cysteine 387(C387) residue of DprE1 enzyme, which catalyze biosynthesis of arabinose. Formation of covalent and non-covalent bond by the interacting ligand with the enzyme causes loss of its catalytic activity which ultimately leads to the death of mycobacteria.

The Benzothiazinone derivatives kills the mycobacterium by inhibiting the essential flavoenzyme DprE1. This study involves designing of a series of 16 benzothiazinone derivatives that binds non-covalently to DprE1. These derivatives were subjected to energy minimization and molecular docking studies with Schrodinger software, from which the binding free energy calculations showed that the suggested compounds had better binding affinity with DprE1.

All the compounds were synthesized by conventional method. The synthesis was done under standard condition using AR grade reagents and the reaction progress was monitored by TLC. Structural conformation was done by IR spectroscopy, NMR and Mass spectroscopy. The synthesized compounds were evaluated for their anti-microbial and anti-tubercular activity against different strains. Out of 5 evaluated compounds 3 compounds exhibited potent activity against microbial (Staphylococcus Aureus and Escherichia Coli) and tubercular (H37RV) strains. BTZ4 and BTZ10 has shown good activity against anti-microbial strain and BTZ8 has shown good activity against anti-tubercular strain.

On the basis of docking results, we conclude that the selected new series of benzothiazinone hybrid derivatives BTZ4, BTZ7, BTZ8, BTZ9, BTZ10 act on the Enzyme DprE1, against tuberculosis binding protein with PDB ID- 4NCR, as they may have better binding sites and better energy values, when compared with the standard drugs Ampicillin, Isoniazid and Rifampicin. So it can be taken as best hit molecule and it could be useful for development of more new antimicrobial and antitubercular agents, blocking the mycobacterial cell wall formation. 


\section{Conflict of interest: None}

6. Funding Sources: This research did not receive any specific grant from funding agencies in the public, commercial, or not-for-profit sectors.

7. Acknowledgement: Authors are thankful to Director, Shri G. S. Institute of Technology and Sciences, Indore for providing facilities to carry out this work.

\section{References}

[1] Cousins D.V., Bastida R, Cataldi A, et al, Tuberculosis in seals caused by a novel member of the Mycobacterium tuberculosis complex: Mycobacterium pinnipedii sp. nov., International journal of systematic and evolutionary microbiology, 2003; 53(5): 1305-1314.

[2] Brisson-Noël A, Lecossier D, Nassif X, et al, Rapid diagnosis of tuberculosis by amplification of mycobacterial DNA in clinical samples, The Lancet, 1989; 334(8671): 10691071.

[3] Scanning electron micrograph of Mycobacterium Tuberculosis image. Available from: https://upload.wikimedia. org/wikipedia/ commons/c/cb/ Mycobacterium_tuberculosis.jpg.

[4] problems related to drugs used in treatment of TB Available from: https://healthywa.wa. gov.au/Articles/J_M/Medications-to-treat-tuberculosis.

[5] Newer Research. Available from: https://en.wikipedia. org/wiki/ Tuberculosis \# New_onset.

[6] Schaaf H.S., Thee S, van der Laan L, et al, Adverse effects of oral second-line antituberculosis drugs in children, Expert Opinion on Drug Safety, 2016; 15(10): 1369-81.

[7] Dos Santos Fernandes G.F., Jornada D.H., De Souza P.C., Chin, et al, Current Advances in Antitubercular Drug Discovery: Potent Prototypes and New Targets, Current medicinal chemistry, 2015; 22(27): 3133-61.

[8] Bakshi M.K., Koman R.S., Molecular docking: a review, International Journal of Research in Ayurveda \& Pharmacy, 2011; 2(6): 1746-1751.

[9] Gawad J, Bonde C, Decaprenyl-phosphoryl-ribose 2'-epimerase (DprE1): challenging target for antitubercular drug discovery, Chem Cent J, 2018; 12(1): 72-81.

[10] Shiqi P, Ming Z, Chunying C, Methods for Biological evaluation and applications of antitubercular assays: Pharmaceutical Bioassays, 2007; 39-44. 
[11] Mounyr B, Moulay S, Saad K, Methods for invitro evaluating anti-microbial activity: Journal of Pharmaceutical Analysis, 2016; 71-79.

[12] Xiong L, Chao G, Yao-Jie S, et al, Identification of New series of benzothiazinone derivatives with excellent antitubercular activity and improved pharmacokinetic profiles: RSC Advances, 2018; 8: 11163-11176.

[13] Zeyu T, Qian L, Yang L, Bin W, Shuo, et al, Design, synthesis and antitubercular evaluation of benzothiazinones containing a piperidine moiety: European Journal of Medicinal Chemistry, 2018; 30303-30309.

[14] Laurent R, Matteo M, Daniela B, et al, Discovery and Development of novel Salicylate synthase (MbtI) furanic inhibitors as antitubercular agents: European Journal of Medicinal Chemistry, 2018; 155: 754-763.

[15] Rupesh Vitthalrao C, Mahesh A, Prashant R, et al, An overview of the development of DprE1 inhibitors for combating the menace of Tuberculosis: Journal of Medicinal Chemistry, 2018; 84-898.

[16] Jineetkumar G, Chandrakant B, Decaprenyl-phosphoryl-ribose 2'-epimerase(DprE1): challenging target for antitubercular drugdiscovery, Chemistry Central Journal, 2018; 305-319.

[17] Jéssika de Oliveira V, Marcus Tullius S, Luciana S, Molecular docking studies of benzothiazinone derivatives in the search for new tuberculostatic agents: Research Gate, 2017; 3: 3051-3064.

[18] Jeremie P, Caroline S, Foo S.Y., Structural studies of Mycobacterium tuberculosis DprE1 interacting with its inhibitors: Elsevier, 2017; 22: 1359-1368.

[19] Brecik M, DprE1 is a vulnerable tuberculosis drug target due to its cell wall localization., ACS Chem, 2015; 10: 1631-1636.

[20] Kai Lv, Xuefu Y, Bin W, et al, Identification of Better Pharmacokinetic Benzothiazinone Derivatives as New Antitubercular Agents containing C-2 nitrogen spiro-heterocycle moiety: ACS Medicinal Chemistry, 2015; 576-588.

[21] Lin Lu, Wang B, Mingliang L, et al, Design, synthesis and antitubercular evaluation of benzothiazinones containing triazolothiadiazole moiety: RSC Advances, 2017; 7: 1480-1483.

[22] Crellin P.K., Decaprenylphosphoryl-beta-D-ribose 20-epimerase, the target of benzothiazinones and dinitrobenzamides, is an essential enzyme in Mycobacterium smegmatis: Journal of Applied Chemistry, 2015; 16869-16882. 
[23] Lessem E, Vernon A, Raviglione M.C., New drugs and new regimens for the treatment of tuberculosis: review of the drug development pipeline and implications for national programmes, Curr. Opin. Pulm. Med., 2015; 16 (3): 186-193.

[24] Mikusova K, Decaprenyl phosphoryl arabinofuranose, the donor of the D-arabinofuranosyl residues of mycobacterial arabinan, is formed via a two-step epimerization of decaprenylphosphoryl ribose: J. Bacteriol., 2014; 187: 8020-8025.

[25] Ashish K, Degiacomi G, Ribeiro A.L., Bedaquiline: A new drug approved for the treatment of multidrug resistant tuberculosis, Curr. Opin. Pulm. Med., 2014; 54 (4): 1616-1618.

[26] Makarov V, Manina G, Mikusova K, Benzothiazinones kill Mycobacterium tuberculosis by blocking arabinan synthesis: European Journal of Medicinal Chemistry, 2014; 801-804.

[27] Ryan N.J., Loura J.H., Delamanid: First Global Approval Drugs, 2014; 74(9): 1041-1045.

[28] Trefzer C, Gonzalez M.R., Hinner M.J. et al, Benzothiazinones: Prodrugs That Covalently Modify the Decaprenylphosphoryl- $\beta$-D-ribose 2'-epimerase DprE1 of Mycobacterium tuberculosis, J. Am. Chem. Soc., 2010; 132: 13663-13665.

[29] Neres J, Pojer F, Molteni E, et al, Structural Basis for Benzothiazinone-Mediated Killing of Mycobacterium tuberculosis: Sci. Transl. Med., 2012; 4(50): 121-150. 